\title{
Cognitive and emotional impairments in adults with attention-deficit/hyperactivity disorder and cocaine use*
}

\author{
Michael D. Wunderlia; Matthias Vonmoos ${ }^{\mathrm{a}}$; Stefania M. Niedecker ${ }^{\mathrm{a}}$; \\ Lea M. Hulka ${ }^{\mathrm{a}}$; Katrin H. Preller ${ }^{\mathrm{a}}$; Markus R. Baumgartner ${ }^{\mathrm{b}}$; \\ Thomas Kraemer ${ }^{\mathrm{b}}$; Erich Seifritz ${ }^{\mathrm{a}, \mathrm{c}}$; Michael P. Schaub ${ }^{\mathrm{d}}$; \\ Dominique Eich-Höchlia ; Boris B. Quednow ${ }^{\mathrm{a}, \mathrm{c}, \text {,** }}$ \\ ${ }^{a}$ Department of Psychiatry, Psychotherapy, and Psychosomatics, Psychiatric Hospital of the University of \\ Zurich, Lenggstrasse 31, 8032 Zurich, Switzerland \\ ${ }^{b}$ Center of Forensic Hairanalytics, Institute of Forensic Medicine, University of Zurich, Kurvenstrasse 17, 8006 \\ Zurich, Switzerland \\ ${ }^{c}$ Neuroscience Center Zurich, University of Zurich and Swiss Federal Institute of Technology Zurich, \\ Winterthurerstrasse 190, 8057 Zurich, Switzerland \\ ${ }^{d}$ Swiss Research Institute for Public Health and Addiction, Associated Institute at the University of Zurich and \\ WHO Collaborating Centre, Konradstrasse 32, 8031 Zurich, Switzerland
}

\begin{abstract}
Abbreviated running title:
Interaction effect of cocaine and ADHD on cognition
\end{abstract}

* Supplementary material can be found by accessing the online version of this paper at http://dx.doi.org and by entering doi:...

\section{**Corresponding author:}

Boris B. Quednow

Experimental and Clinical Pharmacopsychology

Department of Psychiatry, Psychotherapy and Psychosomatics

Psychiatric Hospital of the University of Zurich

Lenggstrasse 31

CH-8032 Zurich, Switzerland

Tel.: 0041-44-384-2777 Fax: 0041-44-384-3396

E-mail: quednow@bli.uzh.ch 


\section{ABSTRACT}

Background: Attention-deficit/hyperactivity disorder (ADHD) is an important modulator of cognitive and social functioning in cocaine addiction but it is unclear whether ADHD symptoms and cocaine use display mutually aggravating interaction effects on cognition, social functioning and depressive symptoms. Therefore, we investigated the interaction of cocaine use and adult ADHD on social and non-social cognition and depressive symptoms. Methods: Twenty-four cocaine users with (CU+ADHD) and 30 without ADHD (CU-ADHD), 29 cocaine-naïve ADHD patients, and 40 cocainenaïve healthy controls underwent comprehensive neuropsychological testing including assessment of social cognition (cognitive/emotional empathy and Theory-of-Mind). Additionally, depressive symptoms were measured with the Beck Depression Inventory. Results: The effect size of global cognitive impairment was largest in CU+ADHD ( $\mathrm{d}=1.22$ vs. controls) followed by CU-ADHD $(\mathrm{d}=0.74)$, and cocaine-naïve ADHD patients $(\mathrm{d}=0.33)$. A similar pattern appeared regarding depressive symptoms (CU+ADHD: $\mathrm{d}=1.47$; CU-ADHD: $\mathrm{d}=0.49$, ADHD: $\mathrm{d}=0.34)$. In the measures of Theory-ofMind (CU+ADHD: d=0.76; CU-ADHD: $d=0.06$, ADHD: $d=0.01$ ) and cognitive empathy (CU+ADHD: $\mathrm{d}=0.80$; CU-ADHD: $\mathrm{d}=0.39$, ADHD: $\mathrm{d}=-0.11$ ) only $\mathrm{CU}+\mathrm{ADHD}$ showed moderate to large impairments. Moreover, two-way analyses of covariance revealed a significant interaction effect of the factors ADHD and cocaine use on depressive symptoms $(p<.05)$ and Theory-of-Mind $(p<.05)$ but not on global cognitive performance ( $p=.64)$. Conclusions: When occurring together, cognitive impairments associated with both ADHD and cocaine use are largely additive, whereas both factors seem to mutually potentiate one another with respect to mood and mental perspective-taking disturbances. Given the high comorbidity between ADHD and cocaine use, longitudinal studies are needed to investigate the origin of these potentiated impairments.

KEYWORDS: stimulants, depression, affective disorder, mentalizing, emotion recognition, chronic use 


\section{INTRODUCTION}

With an estimated 17 million past-year users cocaine remains one of the most used illicit drugs worldwide (United Nations Office on Drugs and Crime, 2015). Because of its negative health consequences and addictive potential, cocaine use represents a major issue in public health (Nutt et al., 2007). Attention-deficit/hyperactivity disorder (ADHD) is another major public health issue (Ballon et al., 2015), with an estimated worldwide prevalence of about 5\% in children (Polanczyk et al., 2014) and symptoms that persist into adulthood in up to $65 \%$ of patients (Faraone et al., 2006). At more than $20 \%$, the prevalence of adult ADHD appears to be much higher in individuals with cocaine use disorder compared with the general population (Perez de Los Cobos et al., 2011; van Emmerik-van Oortmerssen et al., 2012; Vonmoos et al., 2013a). Furthermore, in a sample of adult patients seeking treatment for cocaine addiction, 35\% were found to have ADHD (Lambert and Hartsough, 1998). These numbers are in line with the assumption that adolescents with ADHD are about twice as likely as healthy individuals to develop a substance use disorder (Biederman et al., 1995).

Recent findings from the Zurich Cocaine Cognition Study $\left(\mathrm{ZuCo}^{2} \mathrm{St}\right)$ confirmed that recreational and dependent cocaine users displayed considerable impairments in attention, working memory, declarative memory, and executive functions that were aggravated with increased use (Vonmoos et al., 2013a). Furthermore, recreational and dependent cocaine users showed less emotional empathy, and specifically dependent users displayed difficulties in mental and emotional perspective-taking (also called "mentalizing" or "Theory-of-Mind"), higher delay aversion, and decreased planning abilities (Hulka et al., 2014; Preller et al., 2014). In the $\mathrm{ZuCo}^{2} \mathrm{St}$, social and nonsocial cognition were strongly moderated by comorbid ADHD symptoms, since the combination of cocaine use and ADHD symptoms was associated with much more pronounced deficits (Preller et al., 2014; Vonmoos et al., 2013a).

Cocaine use appears to impact neurotransmitter systems in brain regions thought to be altered in ADHD patients: Chronic cocaine use has been linked to alterations in the fronto-striatal dopamine system (Beveridge et al., 2008; Garavan and Hester, 2007; Volkow et al., 2009a, 2004) and noradrenergic changes in the thalamus and locus coeruleus (Ding et al., 2010). Moreover, structural and functional changes in several areas of the prefrontal cortex have been linked to cognitive deficits 
in dependent cocaine users (Beveridge et al., 2008; Garavan and Hester, 2007; Goldstein et al., 2004). Remarkably, fronto-striatal dysfunctions and changes in catecholaminergic neurotransmitter systems appear to also play a crucial role in the etiology of ADHD (Brennan and Arnsten, 2008; Del Campo et al., 2011; Tripp and Wickens, 2009). In particular, disturbances in cognitive functions such as vigilance, working memory, planning, and response inhibition —as well as problems in motivational processes, such as delay aversion - are associated with both ADHD (Nigg, 2005; Willcutt et al., 2005) and cocaine use (Hulka et al., 2014; Vonmoos et al., 2013a, 2013b). In both cases, these effects have been proposed to depend on changes in the dopamine and noradrenaline system (Gould et al., 2014; Sofuoglu, 2010; Tripp and Wickens, 2009). Recently, also problems in more complex cognitive functions such as social cognition and interaction have been demonstrated in recreational and dependent cocaine users as well as in patients with ADHD (Bora and Pantelis, 2016; Hulka et al., 2014; 2013; Preller et al., 2014). Additionally, both, patients with cocaine addiction and with ADHD have an increased risk for developing depressive symptoms (Connor et al., 2003; Rounsaville, 2004; Swendsen and Merikangas, 2000).

To our knowledge, the interaction of ADHD symptoms and cocaine use with regard to cognitive and socio-cognitive functions as well as to depressive symptoms has not been investigated in detail yet. Thus, it remains unclear whether the pronounced cognitive and socio-cognitive impairments of cocaine users with ADHD symptoms arise from a combination of ADHD and cocaine use or can be explained by ADHD alone (Preller et al., 2014; Vonmoos et al., 2013a). Therefore, we recruited a group of ADHD patients without illegal drug use and compared them with cocaine users with and without ADHD and to stimulant-naïve healthy controls so as to investigate the presumed interactions between ADHD and cocaine use. We hypothesized that ADHD and cocaine use would reveal cumulative or even multiplicative effects.

\section{METHODS}

\subsection{Participants}

We recruited 29 ADHD patients who reported no illegal drug use, 24 cocaine users with ADHD (CU+ADHD), 30 cocaine users without ADHD (CU-ADHD), and 40 stimulant-naïve healthy 
controls and matched the groups for age and sex (see Supplementary Methods $\mathrm{S} 1^{1}$ for recruitment details). All participants had to be between 18 and 60 years old and fluent in German. Exclusion criteria for all participants were current or previous neurological disorders or head injury, any clinically significant medical disease, a family history of schizophrenia or bipolar disorder, and the use of prescription drugs affecting the central nervous system (except for methylphenidate and dexamphetamine for the ADHD group) as well as a lifetime history of opioid use. For controls and ADHD patients, further exclusion criteria were any Axis-I DSM-IV psychiatric disorder (with the exception of ADHD), any form of addiction (except nicotine), and regular illegal drug use (lifetime use $>15$ occasions, except cannabis). Specific exclusion criteria for the cocaine user groups were polytoxic drug use, any Axis-I DSM-IV adult psychiatric disorder (other than ADHD in CU+ADHD) with exception of cocaine, nicotine, and alcohol abuse/dependence and history of depression (acute major depression was excluded). Inclusion criteria for the cocaine user groups were cocaine use of at least $0.5 \mathrm{~g} / \mathrm{month}$, cocaine as the preferred illegal drug, and a current abstinence period of less than 6 months. All participants were asked to abstain from illegal substances for at least three days and from alcohol for at least $24 \mathrm{~h}$ prior to testing. Compliance was controlled by urine toxicology, and selfreported drug use was controlled by a 6-month hair testing (see Supplementary Methods S2). Of the 29 cocaine-naïve ADHD patients, 24 received stimulant treatment prior to the study (23 participants received methylphenidate, 1 participant received dexamphetamine) while four patients showed no history of stimulant medication. ADHD patients were asked not to use prescription stimulants or any other medication for $24 \mathrm{~h}$ prior to testing. The study was approved by the Cantonal Ethics Committee of Zurich, and all participants gave written informed consent and were compensated for their participation.

\subsection{Clinical Assessment}

${ }^{1}$ Supplementary material can be found by accessing the online version of this paper at http://dx.doi.org and by entering doi:... 
Trained psychologists conducted the Structured Clinical Interview for Axis-I DSM-IV disorders in order to exclude participants with an Axis-I DSM-IV psychiatric disorder. Drug use was assessed with the Interview for Psychotropic Drug Consumption (Quednow et al., 2004). ADHD diagnoses and current severity of ADHD symptoms were evaluated with the ADHD self-rating scale (ADHD-SR) (Rosler et al., 2004) corresponding to DSM-IV criteria. Furthermore, the German short version of the Wender Utah Rating Scale (WURS-k) measuring ADHD symptoms present in childhood was used in the ADHD sample (Retz-Junginger et al., 2002). Depressive symptoms - as an outcome measure - were assessed with the Beck Depression Inventory (BDI) (Beck et al., 1961). Premorbid verbal intelligence was estimated with a German vocabulary test (MehrfachwahlWortschatz-Intelligenztest) (Lehrl et al., 1995). Severity of tobacco dependence was measured by the Fagerström Test of Nicotine Dependence (Heatherton et al., 1991). Finally, to measure present cocaine craving in cocaine users, the brief version of the cocaine craving questionnaire (CCQ) was applied (Sussner et al., 2006).

\subsection{Neuropsychological Assessment}

Cognitive performance was assessed with four tests from the Cambridge Neuropsychological Test Automated Battery (Strauss et al., 2006): Rapid Visual Information Processing, Spatial Working Memory, Intra/Extra-Dimensional Set Shifting, and Paired Associates Learning. Additionally, a German version of the Rey Auditory Verbal Learning Test (Helmstaedter et al., 2001) and the Letter Number Sequencing Test were administered (Wechsler, 1997). As previously published (Vonmoos et al., 2013a, 2014), 15 predefined test parameters underwent z-transformation on the basis of means and standard deviations of the control group and were combined into four cognitive domains (Goldstein et al., 2004; Jovanovski et al., 2005; Pace-Schott et al., 2008; Vonmoos et al., 2013a; Woicik et al., 2009): attention, working memory, declarative memory, and executive functions (see Supplementary Methods S3 for details ${ }^{2}$ ). These four domains were equally integrated into a global cognitive index

\footnotetext{
${ }^{2}$ Supplementary material can be found by accessing the online version of this paper at
} $\underline{\text { http://dx.doi.org and by entering doi:... }}$ 
(GCI). To avoid the accumulation of alpha-errors, we focused our analysis on these four domains and the GCI. However, we reported single neuropsychological test scores in the Supplementary Material (Supplementary Table $\mathrm{S}^{3}$ ). Two aspects of social cognition-Theory-of-Mind and empathy-were assessed with the Movie for the Assessment of Social Cognition (MASC) (Dziobek et al., 2006) and the Multifaceted Empathy Test (MET) (Dziobek et al., 2008), both of which have been described in detail in our previous work (see also Supplementary Methods S4 ${ }^{4}$ ) (Preller et al., 2014).

\subsection{Statistical Analysis}

We performed the statistical analyses with SPSS 22.0 for Windows. Demographic and drug use data for all groups were analyzed with Pearson's chi-square test and analyses of variance (ANOVA) followed by Sidak-corrected post-hoc comparisons where appropriate. To investigate group differences over all groups in cognitive and emotional parameters, we performed analysis of covariance (ANCOVA) or multivariate analysis of covariance (MANCOVA) followed by Sidakcorrected post-hoc comparisons. To investigate the interaction between cocaine use and ADHD, we analyzed the data with two-way ANCOVA using the fixed factors of "ADHD" and "cocaine use" (both yes/no). Following significant interaction effects, simple effects were calculated. The significance threshold was set at $p<.05$. To examine if cognitive performance and depressive symptoms were associated with craving for cocaine, Pearson's product moment correlations between relevant outcome measures and the CCQ sum score were calculated (Supplementary Table S2 ${ }^{5}$ ). In order to avoid alpha-error accumulation, the significance threshold was set to $p<.01$ for correlation analyses. Because age is a common confounding variable in investigations of cognition (and especially social cognition), it was introduced as a covariate (Horning et al., 2012; Verhaeghen and Salthouse,

${ }^{3}$ Supplementary material can be found by accessing the online version of this paper at http://dx.doi.org and by entering doi:...

${ }^{4}$ Supplementary material can be found by accessing the online version of this paper at http://dx.doi.org and by entering doi:...

${ }^{5}$ Supplementary material can be found by accessing the online version of this paper at http://dx.doi.org and by entering doi:... 
1997). Because of significant group differences, years of education (YoE) was introduced as a further covariate. We have previously shown that the moderating effect of the co-factor ADHD on global cognitive performance in cocaine users was large with an effect size of Cohen's $f=0.49$ and a partial eta-square $=0.195$ (Vonmoos et al., 2013a). In an a priori power analysis, we therefore assumed a lower effect size of $\mathrm{f}=0.40$, an $\alpha$-error probability of $5 \%$, and a conservative power of $90 \%$ for the present ANCOVA design with 4 groups and 2 covariates, suggesting a total minimum sample size of 93 individuals.

\section{RESULTS}

\subsection{Demographic Characteristics and Drug Use}

The groups did not differ significantly in age and sex distribution (Table 1). However, the groups significantly differed in YoE and verbal IQ: CU+ADHD had fewer YoE and lower verbal IQ scores than controls and ADHD patients. As expected, the four groups differed in ADHD symptom severity as measured by the ADHD-SR. The ADHD group reported the most severe ADHD symptom scores, which differed significantly from controls and CU-ADHD but importantly did not differ significantly from CU+ADHD ( $p=.49)$. On the WURS-k, the ADHD group reported a mean score of $36.8 \pm 15.9$ (SD), which is above the diagnostic cut-off of 30 points (Retz-Junginger et al., 2002).

Notably, CU-ADHD did not differ significantly from CU+ADHD for any self-reported cocaine use parameter or for frequency of DSM-IV cocaine dependence (Table 1). Moreover, CU+ADHD and CUADHD showed no significant differences regarding cocaine hair concentrations or frequency of cocaine-positive urine testings. Accordingly, CU-ADHD and CU+ADHD did also not differ significantly in the CCQ sum score. In addition, correlation analysis showed that test performance and depressive symptom scores did not correlate with craving for cocaine (Supplementary Table S2). Hair testings confirmed a clear preference for cocaine versus other drugs for both groups (Supplementary Table S3) and revealed the highest methylphenidate concentrations in ADHD patients (Table 1). CU+ADHD and CU-ADHD smoked significantly more cigarettes than the ADHD patients, and CU+ADHD also smoked more frequently than controls. Both cocaine user groups reported greater weekly alcohol consumption than the ADHD group. 


\subsection{Cognition}

One-way ANCOVAs (with YoE and age as covariates) performed for the GCI and the four cognitive domains revealed significant group differences in the $\mathrm{GCI}(\mathrm{F}(3,117)=12.12, p<.001)$, working memory $(\mathrm{F}(3,117)=8.87, p<.001)$, declarative memory $(\mathrm{F}(3,117)=14.07, p<.001)$, executive functions $(\mathrm{F}(3,117)=2.68, p<.05)$, and the attention domain score $(\mathrm{F}(3,117)=4.16, p<.01)$. In addition, clear linear trends (controls>ADHD>CU-ADHD>CU+ADHD) were found $(p<.001-.01)$ in all cognitive domains (Fig. 1).

Sidak post-hoc comparisons revealed significant differences between controls and both cocaine-using groups in the GCI, working memory, and declarative memory ( $p<.001-.01)$, indicating cognitive impairments in both user groups. CU+ADHD also differed from controls in attention $(p<.01)$ and performed worse than the ADHD group in the GCI and the two memory domains $(p<.001-.01)$. CU-ADHD performed better than CU+ADHD for declarative memory $(p<.05)$ (Fig. 1). ADHD patients showed no significant performance deficits compared with the controls in any of the four domains or the GCI.

A two-way ANCOVA revealed significant main effects of $\operatorname{ADHD}(\mathrm{F}(1,117)=7.62, p<.01)$ and cocaine use $(\mathrm{F}(1,117)=28.49, p<.001)$ on the GCI but no significant interaction effect $(\mathrm{F}(1,117)=.21$, $p=.64$ )(Fig. 2a). Similar effects were found for the other domains-except for attention and executive functions, for which the main effects for ADHD were not significant $(\mathrm{F}(1,117)=2.58$ and $0.61, p=.11$ and .44 , respectively).

\subsection{Social cognition}

The MANCOVA (with YoE and age as covariates) performed for the z-transformed variables cognitive empathy (CE), explicit emotional empathy (EEE), and implicit emotional empathy (IEE) on the MET showed a significant main effect for group using Hotelling's trace $(\mathrm{V}=.21, \mathrm{~F}(9,341)=2.66$, $p<.01)$. Groups differed significantly in $\mathrm{CE}(\mathrm{F}(3,117)=4.62, p<.01)$. In addition, linear trends (controls $>$ ADHD $>$ CU-ADHD $>$ CU+ADHD) were found $(p<.001-.05)$ for all of the three MET variables. For Theory-of-Mind, an ANCOVA revealed significant group differences $(F(3,117)=3.68$, 
$p<.05)$ in the MASC sum score (Supplementary Fig. $\left.\mathrm{S} 1^{6}\right)$ and a significant linear trend $(p<.01$, controls $>$ ADHD $>$ CU-ADHD $>$ CU+ADHD). Sidak post-hoc comparisons revealed that CU+ADHD made more errors than controls and ADHD patients in CE and Theory-of-Mind $(p<.01-.05)$ and that CU-ADHD performed better than CU+ADHD on the MASC ( $p<.05)$. Analysis of the different MASC error types are shown in the Supplementary Material (Supplementary Fig. S2 ${ }^{7}$ ). Notably, ANCOVA for overmentalizing errors (excessive Theory-of-Mind) yielded a significant main effect for group $(\mathrm{F}(3,117)=4.08, p<.01)$, and Sidak post-hoc comparisons revealed significant differences between CU+ADHD and ADHD patients and between CU+ADHD and controls $(p<.01$ and <.05).

Two-way ANCOVAs on the MET scores revealed a significant main effect for cocaine use on $\mathrm{CE}(\mathrm{F}(1,117)=12.65, p<.001)$ and $\operatorname{IEE}(\mathrm{F}(1,117)=7.70, p<.01)$ but not on $\operatorname{EEE}(\mathrm{F}(1,117)=3.61, p=.06)$. For ADHD, no significant main effects were found $(\mathrm{F}(1,117)=0.02-0.85, p=.36-.88)$, and the two factors showed no significant interaction effects $(\mathrm{F}=(1,117)=0.18-2.16, p=.14-.67)$.

The two-way ANCOVA on Theory-of-Mind revealed that the interaction of ADHD and cocaine use had a significant effect on mental and emotional perspective-taking performance $(\mathrm{F}(1,117)=4.09, p<.05)$. The main effects for $\operatorname{ADHD}(\mathrm{F}(1,117)=3.93, p<.05)$ and cocaine use $(\mathrm{F}(1,117)=5.21, p<.05)$ were also significant (Fig. 2b). Simple effect analysis revealed that cocaine users did not differ from non-users in the no ADHD group $(\mathrm{F}(1,117)=0.20, p=.66)$, whereas cocaine use was associated with a significantly worse performance in the ADHD group $(F(1,117)=7.23$, $p<.01)$.

Finally, a significant ADHD*cocaine use interaction effect $(\mathrm{F}(1,117)=4.84, \quad p<.05)$ (Supplementary Fig. $\mathrm{S3}^{8}$ ), as well as a main effect for cocaine on overmentalizing errors

${ }^{6}$ Supplementary material can be found by accessing the online version of this paper at http://dx.doi.org and by entering doi:...

${ }^{7}$ Supplementary material can be found by accessing the online version of this paper at http://dx.doi.org and by entering doi:...

${ }^{8}$ Supplementary material can be found by accessing the online version of this paper at http://dx.doi.org and by entering doi:... 
$(\mathrm{F}(1,117)=8.57, p<.01)$ appeared. The tendency to overmentalize was higher in cocaine users compared to non-users among the participants with $\operatorname{ADHD}(\mathrm{F}(1,117)=10.86, p<.001)$.

\subsection{Depressive Symptoms}

The ANCOVA (with YoE and age as covariates) for the BDI score showed a significant main effect for group $(\mathrm{F}(3,117)=13.82, p<.001)$ as well a significant linear trend (controls $<$ ADHD $<$ CUADHD $<\mathrm{CU}+\mathrm{ADHD})(p<.001)$ (Fig. 3). CU+ADHD showed increased BDI scores versus all three other groups $(p<.001)$. The two-way ANCOVA revealed significant main effects for ADHD $(\mathrm{F}(3,117)=17.33, p<.001)$ and cocaine use $(\mathrm{F}(1,117)=24.24, p<.001)$ and a significant interaction effect $(\mathrm{F}(1,117)=4.06, p<.05)$ (Fig. 2c), indicating an amplification of emotional disturbances in the CU+ADHD group compared with the other three groups. Simple effect analyses showed that cocaine use is associated with significantly higher BDI scores in individuals with $(\mathrm{F}(1,117)=18.47, p<.001)$ and without $\operatorname{ADHD}(\mathrm{F}(1,117)=7.58, p<.01)$.

\section{DISCUSSION}

The aim of the study was to investigate the interaction between ADHD and cocaine use with regard to non-social and social cognition and depressive symptoms. Detailed psychiatric diagnostics and hair toxicology were used to minimize the influence of psychiatric comorbidities and polytoxic drug use. We were able to demonstrate that cocaine users with ADHD symptoms (CU+ADHD) show stronger cognitive impairments than cocaine users without ADHD (CU-ADHD) or ADHD patients without cocaine use. Considering the increase in effect size, our data suggest that the combined detrimental effects of ADHD and cocaine on cognition are largely additive and that the factors seem to potentiate each other regarding depressive symptoms and mentalizing deficits. Thus, we supported our previous assumption that $\mathrm{ADHD}$ and cocaine use might exert mutually aggravating effects on cognitive performance and mental perspective-taking (Preller et al., 2014; Vonmoos et al., $2013 \mathrm{a}$ ). Finally, adult ADHD patients without illicit drug consumption do not display impairments regarding cognitive and emotional empathy or mental and emotional perspective-taking.

Our study supports the hypothesis that the interaction of ADHD and cocaine use has a summative effect on general cognitive performance. Furthermore, we found only small to moderate performance deficits across all cognitive domains $(d=0.17-0.37)$ in our sample of cocaine-naïve adults 
with ADHD. However, in addition to the limited power, rather small effect sizes can be expected in the executive functions of adult ADHD patients because of improved compensation for possible deficits with age (Nigg et al., 2005). Most studies reporting executive function deficits in adult ADHD patients have not assessed comorbidities, which may account for many findings of executive impairments (Nigg et al., 2005). Furthermore, previous findings suggest that declarative memory deficits are common in adult ADHD (Verster et al., 2010), which is in line with the present results: Our ADHD patients showed the strongest, although still not significant, impairment in this domain. Surprisingly, we did not find strong impairments in the attention domain, as might be expected in a disorder defined by attentional deficits. However, the distinction between adult ADHD patients and controls is usually based on omission errors and variability in reaction times in sustained attention and selective tasks over a minimum of 14 minutes (Conners and Sitarenios, 2011; Hervey et al., 2004). By contrast, our attention domain included two parameters of a sustained attention task of a maximum length of 5 minutes and a supraspan parameter of a verbal learning task, which required less than 2 minutes. Furthermore, most of our cocaine-naive ADHD patients received pharmacological treatment with prescription stimulants prior to the study, which might have led to an underestimation of their potential cognitive deficits. However, acute cognitive improvements in adult ADHD patients medicated with methylphenidate should be expected primarily in the attention domain-less so in other cognitive domains (Advokat, 2010). On the other hand, because we asked our ADHD patients to abstain from their daily stimulant medication for at least $24 \mathrm{~h}$ so as to exclude acute drug effects, the beneficial effects of methylphenidate are expected to no longer be present at the time of testing. Moreover, it should be noticed that our ADHD patients but also our cocaine using groups had a high level of functioning as all of the ADHD patients as well as $80 \%$ of the cocaine users were employed.

We have previously shown that dependent and recreational cocaine users display impaired explicit and implicit emotional empathy (Preller et al., 2014). Probably owing to the smaller sample sizes of the present cocaine groups and the mixture of recreational and dependent cocaine users, the present study showed only moderate effect sizes $(d=0.24-0.55)$, which were, however, in the same range as reported in our previous publication (Preller et al., 2014). Furthermore, we previously demonstrated that CU+ADHD but not CU-ADHD individuals show significant mental perspective- 
taking deficits (as measured by MASC; Preller et al., 2014), which is in line with the present results coming from an overlapping sample. Accordingly, the two-way ANCOVA of the MASC sum score revealed that the observed drop in performance in CU+ADHD can be explained by the mutual interaction of cocaine use and ADHD. Interestingly, it is not a general lack of Theory-of-Mind that accounts for group differences but rather the tendency of CU+ADHD to overmentalize. Thus, CU+ADHD might fail to understand the perspective of others because they overinterpret social signs (Preller et al., 2014).

The literature on social cognition in adult ADHD is surprisingly sparse and even in children with ADHD, empathy and Theory-of-Mind have rarely been investigated (Uekermann et al., 2010). However, there is some evidence that empathy and Theory-of-Mind may be affected in children with ADHD (Braaten and Rosen, 2000; Buitelaar et al., 1999; Dyck et al., 2001). Because social cognition is essential for successful social interactions, the adult patients in our sample have perhaps developed strategies to compensate for possible social cognitive deficits. This may explain the lack of performance differences in social cognition in comparison to controls.

Regarding depressive symptoms, CU+ADHD displayed higher BDI scores than the controls, the ADHD patients, and CU-ADHD, indicating increased emotional burdens in CU+ADHD. Furthermore, a significant interaction effect indicated that cocaine use and ADHD mutually potentiate depressive symptoms. This finding is in line with the frequent comorbidity between depression and ADHD, as well as between depression and substance use disorders (Abraham and Fava, 1999; Daviss, 2008; Swendsen and Merikangas, 2000). Moreover, there is evidence for a relationship between depression and trait impulsivity (Swann et al., 2008), a core feature of ADHD (Wilson, 2007) that is also elevated in cocaine users (Vonmoos et al., 2013b).

As mentioned in the Introduction, there are potential commonalities between ADHD and cocaine use at the level of their neurobiological basis. The majority of imaging studies describes increased dopamine transporter density; decreased dopamine $\mathrm{D}_{2}$ receptor availability; and decreased dopamine synthesis, storage, and release in both ADHD patients and chronic cocaine users (Trifilieff and Martinez, 2013; Zimmer, 2009). However, some conflicting results have been found regarding dopamine transporter density and the availability of $\mathrm{D}_{2}$ receptors in ADHD patients (Volkow et al., 
2009b), whereas for chronic cocaine users, the results seem to be more consistent. Additionally, recent data from our lab suggest that cognitive impairments in cocaine users are likely drug-induced (Vonmoos et al., 2014), whereas cognitive dysfunctions in ADHD should be inherent clinical features of the syndrome (Biederman, 2005). We therefore propose that cocaine-induced neurochemical adaptations amplify the ADHD-related abnormalities of the monoamine neurotransmitter systems (Tripp and Wickens, 2009). Although cocaine seems to deteriorate further the cognitive abilities of ADHD patients, the stimulant methylphenidate, which is used to target the cognitive problems of ADHD patients, has not been shown to lead to such an interaction (yet). This might be explained by the different pharmacokinetics of these two substances. The strong cocaine-induced peaks of neurotransmitter release (Nestler, 2005) and the fast clearance of the drug seem to be more problematic with regard to neurochemical changes and associated cognitive dysfunctions than the slower brain uptake and clearance for (orally consumed) methylphenidate (Volkow et al., 1995, 1998).

The current study has some limitations. Recent findings suggest that a single factor may account for interindividual variance in general psychopathology (e.g., Caspi's p-factor; Caspi et al., 2014). Thus, the members of the CU+ADHD group might be more fraught in terms of general psychopathology than the CU-ADHD or ADHD patients. Because it is impossible to rule out this possibility in a study with a cross-sectional design, longitudinal investigations are needed on the interaction of cocaine use and ADHD. Furthermore, most of the ADHD patients in this study were previously medicated with prescription stimulants, whereas the CU+ADHD individuals were not (see above). However, at the test session they were abstinent for at least $24 \mathrm{~h}$. Thus, further investigations on the interaction of ADHD and cocaine should focus on unmedicated ADHD patients. Finally, the CU+ADHD displayed a more severe pattern of substance use specifically with regard to cannabis. Thus, it is not fully clear how cannabis use interacts with cocaine and ADHD on cognition. However, all relevant effect sizes remained the same if weekly cannabis use was introduced as a further covariate.

In conclusion, our data indicate that cognitive and social deficits are considerably worse in cocaine users with ADHD in comparison to cocaine users without ADHD and to ADHD patients without cocaine use. The cognitive impairments of ADHD and cocaine use seem to be additive, 
whereas cocaine use and adult ADHD appear to mutually amplify emotional disturbances and perspective-taking deficits in cocaine users with ADHD. Given the considerable comorbidity between ADHD and cocaine use disorder (Katusic et al., 2005; Wilens, 2007), longitudinal studies are needed to investigate the causal relationship behind the interaction of cocaine use and ADHD. Additionally, the impact of substances such as tobacco, alcohol and cannabis on cognitive and socio-cognitive deficits as well as on depressive symptoms need further investigation specifically in individuals with ADHD and cocaine use. Finally, the present data strongly suggest that ADHD patients should be better informed about their specific cognitive and emotional risks of cocaine abuse. 


\section{REFERENCES}

Abraham, H.D., Fava, M., 1999. Order of onset of substance abuse and depression in a sample of depressed outpatients. Compr. Psychiatry 40, 44-50.

Advokat, C., 2010. What are the cognitive effects of stimulant medications? Emphasis on adults with attention-deficit/hyperactivity disorder (ADHD). Neurosci. Biobehav. Rev. 34, 1256-1266.

Ballon, N., Brunault, P., Cortese, S., 2015. Sensation seeking and cocaine dependence in adults with reported childhood ADHD. J Atten. Disord. 19, 335-342.

Beck, A.T., Ward, C.H., Mendelson, M., Mock, J., Erbaugh, J., 1961. An inventory for measuring depression. Arch. Gen. Psychiatry 4, 561-571.

Beveridge, T.J., Gill, K.E., Hanlon, C.A., Porrino, L.J., 2008. Review. Parallel studies of cocainerelated neural and cognitive impairment in humans and monkeys. Philos. Trans. R. Soc. Lond. B Biol. Sci. 363, 3257-3266.

Biederman, J., 2005. Attention-deficit/hyperactivity disorder: a selective overview. Biol. Psychiatry $57,1215-1220$.

Biederman, J., Wilens, T., Mick, E., Milberger, S., Spencer, T.J., Faraone, S.V., 1995. Psychoactive substance use disorders in adults with attention deficit hyperactivity disorder (ADHD): effects of ADHD and psychiatric comorbidity. Am. J. Psychiatry 152, 1652-1658.

Bora, E., Pantelis, C., 2016. Meta-analysis of social cognition in attention-deficit/hyperactivity disorder (ADHD): comparison with healthy controls and autistic spectrum disorder. Psychol. Med. 46, 699-716.

Braaten, E.B., Rosen, L.A., 2000. Self-regulation of affect in attention deficit-hyperactivity disorder (ADHD) and non-ADHD boys: differences in empathic responding. J. Consult. Clin. Psychol. 68, 313321.

Brennan, A.R., Arnsten, A.F., 2008. Neuronal mechanisms underlying attention deficit hyperactivity disorder: the influence of arousal on prefrontal cortical function. Ann. N. Y. Acad. Sci. 1129, 236-245. Buitelaar, J.K., van der Wees, M., Swaab-Barneveld, H., van der Gaag, R.J., 1999. Theory of mind and emotion-recognition functioning in autistic spectrum disorders and in psychiatric control and normal children. Dev. Psychopathol. 11, 39-58. 
Caspi, A., Houts, R.M., Belsky, D.W., Goldman-Mellor, S.J., Harrington, H., Israel, S., Meier, M.H., Ramrakha, S., Shalev, I., Poulton, R., Moffitt, T.E., 2014. The p Factor: one general psychopathology factor in the structure of psychiatric disorders? Clin. Psychol. Sci. 2, 119-137.

Conners, C.K., Sitarenios, G., 2011. Conners' Continuous Performance Test (CPT) In: Kreutzer, J., DeLuca, J., Caplan, B. (Eds.), Encyclopedia of Clinical Neuropsychology. Springer, New York, pp. 681-683.

Connor, D.F., Edwards, G., Fletcher, K.E., Baird, J., Barkley, R.A., Steingard, R.J., 2003. Correlates of comorbid psychopathology in children with ADHD. J. Am. Acad. Child Adolesc. Psychiatry 42, 193-200.

Daviss, W.B., 2008. A review of co-morbid depression in pediatric ADHD: etiology, phenomenology, and treatment. J. Child Adolesc. Psychopharmacol. 18, 565-571.

Del Campo, N., Chamberlain, S.R., Sahakian, B.J., Robbins, T.W., 2011. The roles of dopamine and noradrenaline in the pathophysiology and treatment of attention-deficit/hyperactivity disorder. Biol. Psychiatry 69, e145-157.

Ding, Y.S., Singhal, T., Planeta-Wilson, B., Gallezot, J.D., Nabulsi, N., Labaree, D., Ropchan, J., Henry, S., Williams, W., Carson, R.E., Neumeister, A., Malison, R.T., 2010. PET imaging of the effects of age and cocaine on the norepinephrine transporter in the human brain using $(\mathrm{S}, \mathrm{S})-[(11) \mathrm{C}] \mathrm{O}-$ methylreboxetine and HRRT. Synapse 64, 30-38.

Dyck, M.J., Ferguson, K., Shochet, I.M., 2001. Do autism spectrum disorders differ from each other and from non-spectrum disorders on emotion recognition tests? Eur. Child Adolesc. Psychiatry 10, 105-116.

Dziobek, I., Fleck, S., Kalbe, E., Rogers, K., Hassenstab, J., Brand, M., Kessler, J., Woike, J.K., Wolf, O.T., Convit, A., 2006. Introducing MASC: a movie for the assessment of social cognition. J. Autism Dev. Disord. 36, 623-636.

Dziobek, I., Rogers, K., Fleck, S., Bahnemann, M., Heekeren, H.R., Wolf, O.T., Convit, A., 2008. Dissociation of cognitive and emotional empathy in adults with Asperger syndrome using the Multifaceted Empathy Test (MET). J. Autism Dev. Disord. 38, 464-473. 
Faraone, S.V., Biederman, J., Mick, E., 2006. The age-dependent decline of attention deficit hyperactivity disorder: a meta-analysis of follow-up studies. Psychol. Med. 36, 159-165.

Garavan, H., Hester, R., 2007. The role of cognitive control in cocaine dependence. Neuropsychol. Rev. 17, 337-345.

Goldstein, R.Z., Leskovjan, A.C., Hoff, A.L., Hitzemann, R., Bashan, F., Khalsa, S.S., Wang, G.J., Fowler, J.S., Volkow, N.D., 2004. Severity of neuropsychological impairment in cocaine and alcohol addiction: association with metabolism in the prefrontal cortex. Neuropsychologia 42, 1447-1458. Gould, R.W., Duke, A.N., Nader, M.A., 2014. PET studies in nonhuman primate models of cocaine abuse: translational research related to vulnerability and neuroadaptations. Neuropharmacology 84 , $138-151$.

Heatherton, T.F., Kozlowski, L.T., Frecker, R.C., Fagerstrom, K.O., 1991. The Fagerstrom Test for Nicotine Dependence: a revision of the Fagerstrom Tolerance Questionnaire. Br. J. Addict. 86, 11191127.

Helmstaedter, C., Lendt, M., Lux, S., 2001. Verbaler Lern- und Merkfähigkeitstest. Beltz, Göttingen. Hervey, A.S., Epstein, J.N., Curry, J.F., 2004. Neuropsychology of adults with attentiondeficit/hyperactivity disorder: a meta-analytic review. Neuropsychology 18, 485-503. Horning, S.M., Cornwell, R.E., Davis, H.P., 2012. The recognition of facial expressions: an investigation of the influence of age and cognition. Neuropsychol. Dev. Cogn. B Aging Neuropsychol. Cogn. 19, 657-676.

Hulka, L.M., Eisenegger, C., Preller, K.H., Vonmoos, M., Jenni, D., Bendrick, K., Baumgartner, M.R., Seifritz, E., Quednow, B.B., 2014. Altered social and non-social decision-making in recreational and dependent cocaine users. Psychol. Med. 44, 1015-1028.

Hulka, L.M., Preller, K.H., Vonmoos, M., Broicher, S.D., Quednow, B.B., 2013. Cocaine users manifest impaired prosodic and cross-modal emotion processing. Front. Psychiatry 4, 98. Jovanovski, D., Erb, S., Zakzanis, K.K., 2005. Neurocognitive deficits in cocaine users: a quantitative review of the evidence. J. Clin. Exp. Neuropsychol. 27, 189-204.

Katusic, S.K., Barbaresi, W.J., Colligan, R.C., Weaver, A.L., Leibson, C.L., Jacobsen, S.J., 2005. Psychostimulant treatment and risk for substance abuse among young adults with a history of 
attention-deficit/hyperactivity disorder: a population-based, birth cohort study. J. Child Adolesc. Psychopharmacol. 15, 764-776.

Lambert, N.M., Hartsough, C.S., 1998. Prospective study of tobacco smoking and substance dependencies among samples of ADHD and non-ADHD participants. J. Learn. Disabil. 31, 533-544. Lehrl, S., Triebig, G., Fischer, B., 1995. Multiple choice vocabulary test MWT as a valid and short test to estimate premorbid intelligence. Acta Neurol. Scand. 91, 335-345.

Nestler, E.J., 2005. The neurobiology of cocaine addiction. Sci. Pract. Perspect. 3, 4-10.

Nigg, J.T., 2005. Neuropsychologic theory and findings in attention-deficit/hyperactivity disorder: the state of the field and salient challenges for the coming decade. Biol. Psychiatry 57, 1424-1435.

Nigg, J.T., Stavro, G., Ettenhofer, M., Hambrick, D.Z., Miller, T., Henderson, J.M., 2005. Executive functions and ADHD in adults: evidence for selective effects on ADHD symptom domains. J. Abnorm. Psychol. 114, 706-717.

Nutt, D., King, L.A., Saulsbury, W., Blakemore, C., 2007. Development of a rational scale to assess the harm of drugs of potential misuse. Lancet 369, 1047-1053.

Pace-Schott, E.F., Morgan, P.T., Malison, R.T., Hart, C.L., Edgar, C., Walker, M., Stickgold, R., 2008. Cocaine users differ from normals on cognitive tasks which show poorer performance during drug abstinence. Am. J. Drug Alcohol Abuse. 34, 109-121.

Perez de Los Cobos, J., Sinol, N., Puerta, C., Cantillano, V., Lopez Zurita, C., Trujols, J., 2011. Features and prevalence of patients with probable adult attention deficit hyperactivity disorder who request treatment for cocaine use disorders. Psychiatry Res. 185, 205-210.

Polanczyk, G.V., Willcutt, E.G., Salum, G.A., Kieling, C., Rohde, L.A., 2014. ADHD prevalence estimates across three decades: an updated systematic review and meta-regression analysis. Int. J. Epidemiol. 43, 434-442.

Preller, K.H., Hulka, L.M., Vonmoos, M., Jenni, D., Baumgartner, M.R., Seifritz, E., Dziobek, I., Quednow, B.B., 2014. Impaired emotional empathy and related social network deficits in cocaine users. Addict. Biol. 19, 452-466. 
Quednow, B.B., Kuhn, K.U., Hoenig, K., Maier, W., Wagner, M., 2004. Prepulse inhibition and habituation of acoustic startle response in male MDMA ('ecstasy') users, cannabis users, and healthy controls. Neuropsychopharmacology 29, 982-990.

Retz-Junginger, P., Retz, W., Blocher, D., Weijers, H.G., Trott, G.E., Wender, P.H., Rossler, M., 2002. Wender Utah rating scale. The short-version for the assessment of the attention-deficit hyperactivity disorder in adults. Nervenarzt. 73, 830-838.

Rosler, M., Retz, W., Retz-Junginger, P., Thome, J., Supprian, T., Nissen, T., Stieglitz, R.D., Blocher, D., Hengesch, G., Trott, G.E., 2004. Tools for the diagnosis of attention-deficit/hyperactivity disorder in adults. Self-rating behaviour questionnaire and diagnostic checklist. Nervenarzt. 75, 888-895. Rounsaville, B.J., 2004. Treatment of cocaine dependence and depression. Biol. Psychiatry 56, 803809.

Sofuoglu, M., 2010. Cognitive enhancement as a pharmacotherapy target for stimulant addiction. Addiction 105, 38-48.

Strauss, E., Sherman, E.M., Spreen, O., 2006. A Compendium Of Neuropsychological Tests: Administration, Norms, And Commentary. Oxford University Press, Oxford.

Sussner, B.D., Smelson, D.A., Rodrigues, S., Kline, A., Losonczy, M., Ziedonis, D., 2006. The validity and reliability of a brief measure of cocaine craving. Drug Alcohol Depend. 83, 233-237. Swann, A.C., Steinberg, J.L., Lijffijt, M., Moeller, F.G., 2008. Impulsivity: differential relationship to depression and mania in bipolar disorder. J. Affect. Disord. 106, 241-248.

Swendsen, J.D., Merikangas, K.R., 2000. The comorbidity of depression and substance use disorders. Clin. Psychol. Rev. 20, 173-189.

Trifilieff, P., Martinez, D., 2013. Kappa-opioid receptor signaling in the striatum as a potential modulator of dopamine transmission in cocaine dependence. Front. Psychiatry 4, 44. Tripp, G., Wickens, J.R., 2009. Neurobiology of ADHD. Neuropharmacology 57, 579-589. Uekermann, J., Kraemer, M., Abdel-Hamid, M., Schimmelmann, B.G., Hebebrand, J., Daum, I., Wiltfang, J., Kis, B., 2010. Social cognition in attention-deficit hyperactivity disorder (ADHD). Neurosci. Biobehav. Rev. 34, 734-743. 
United Nations Office on Drugs and Crime, 2015. World Drug Report 2015. United Nations

Publication, Vienna.

van Emmerik-van Oortmerssen, K., van de Glind, G., van den Brink, W., Smit, F., Crunelle, C.L., Swets, M., Schoevers, R.A., 2012. Prevalence of attention-deficit hyperactivity disorder in substance use disorder patients: a meta-analysis and meta-regression analysis. Drug Alcohol Depend. 122, 11-19. Verhaeghen, P., Salthouse, T.A., 1997. Meta-analyses of age-cognition relations in adulthood: estimates of linear and nonlinear age effects and structural models. Psychol. Bull. 122, 231-249. Verster, J.C., Bekker, E.M., Kooij, J.J., Buitelaar, J.K., Verbaten, M.N., Volkerts, E.R., Olivier, B., 2010. Methylphenidate significantly improves declarative memory functioning of adults with ADHD. Psychopharmacology (Berl.) 212, 277-281.

Volkow, N.D., Ding, Y.S., Fowler, J.S., Wang, G.J., Logan, J., Gatley, J.S., Dewey, S., Ashby, C., Liebermann, J., Hitzemann, R., et al., 1995. Is methylphenidate like cocaine? Studies on their pharmacokinetics and distribution in the human brain. Arch. Gen. Psychiatry 52, 456-463.

Volkow, N.D., Fowler, J.S., Wang, G.J., Baler, R., Telang, F., 2009a. Imaging dopamine's role in drug abuse and addiction. Neuropharmacology 56 Suppl. 1, 3-8.

Volkow, N.D., Fowler, J.S., Wang, G.J., Swanson, J.M., 2004. Dopamine in drug abuse and addiction: results from imaging studies and treatment implications. Mol. Psychiatry 9, 557-569.

Volkow, N.D., Wang, G.J., Fowler, J.S., Gatley, S.J., Logan, J., Ding, Y.S., Hitzemann, R., Pappas, N., 1998. Dopamine transporter occupancies in the human brain induced by therapeutic doses of oral methylphenidate. Am. J. Psychiatry 155, 1325-1331.

Volkow, N.D., Wang, G.J., Kollins, S.H., Wigal, T.L., Newcorn, J.H., Telang, F., Fowler, J.S., Zhu, W., Logan, J., Ma, Y., Pradhan, K., Wong, C., Swanson, J.M., 2009b. Evaluating dopamine reward pathway in ADHD: clinical implications. JAMA 302, 1084-1091.

Vonmoos, M., Hulka, L.M., Preller, K.H., Jenni, D., Baumgartner, M.R., Stohler, R., Bolla, K.I., Quednow, B.B., 2013a. Cognitive dysfunctions in recreational and dependent cocaine users: role of attention-deficit hyperactivity disorder, craving and early age at onset. Br. J. Psychiatry 203, 35-43. 
Vonmoos, M., Hulka, L.M., Preller, K.H., Jenni, D., Schulz, C., Baumgartner, M.R., Quednow, B.B., 2013b. Differences in self-reported and behavioral measures of impulsivity in recreational and dependent cocaine users. Drug Alcohol Depend. 133, 61-70.

Vonmoos, M., Hulka, L.M., Preller, K.H., Minder, F., Baumgartner, M.R., Quednow, B.B., 2014.

Cognitive impairment in cocaine users is drug-induced but partially reversible: evidence from a longitudinal study. Neuropsychopharmacology 39, 2200-2210.

Wechsler, D., 1997. Wechsler Memory Scale (WMS-III). Psychological Corporation.

Wilens, T.E., 2007. The nature of the relationship between attention-deficit/hyperactivity disorder and substance use. J. Clin. Psychiatry 68 Suppl. 11, 4-8.

Willcutt, E.G., Doyle, A.E., Nigg, J.T., Faraone, S.V., Pennington, B.F., 2005. Validity of the executive function theory of attention-deficit/hyperactivity disorder: a meta-analytic review. Biol. Psychiatry 57, 1336-1346.

Wilson, J.J., 2007. ADHD and substance use disorders: developmental aspects and the impact of stimulant treatment. Am. J. Addict. 16 Suppl. 1, 5-11; quiz 12-13.

Woicik, P.A., Moeller, S.J., Alia-Klein, N., Maloney, T., Lukasik, T.M., Yeliosof, O., Wang, G.J., Volkow, N.D., Goldstein, R.Z., 2009. The neuropsychology of cocaine addiction: recent cocaine use masks impairment. Neuropsychopharmacology 34, 1112-1122.

Zimmer, L., 2009. Positron emission tomography neuroimaging for a better understanding of the biology of ADHD. Neuropharmacology 57, 601-607. 
Table 1: Demographic data and drug use (means and standard deviations)

\begin{tabular}{|c|c|c|c|c|c|c|c|c|c|}
\hline & Controls & ADHD & CU-ADHD & CU+ADHD & $\mathbf{F}$ & $\chi^{2}$ & $t$ test & $d f, d f_{\text {err }}$ & $p$ \\
\hline $\mathrm{n}(\%)$ & $40(32.5)$ & $29(23.5)$ & $30(24.5)$ & $24(19.5)$ & & & & & \\
\hline Age, years & $26.1(5.5)$ & $25.7(6.3)$ & $26.9(6)$ & $27.7(6.6)$ & 0.59 & & & 3,119 & .625 \\
\hline Sex (female/male) & $22 / 18$ & $17 / 12$ & $13 / 17$ & $6 / 18$ & & 7.42 & & 3 & .060 \\
\hline Years of education & $11.5(1.6)$ & $11.7(1.8)$ & $11(1.9)$ & $10.2(1.4)^{* \neq \dagger \dagger}$ & 4.49 & & & 3,119 & .005 \\
\hline Verbal intelligence & $104.3(8.1)$ & $104.6(9.7)$ & $101.6(10)$ & $97.8(7.7)^{* \dagger}$ & 3.39 & & & 3,119 & .020 \\
\hline ADHD-SR & $7.5(5.3)$ & $29.2(10.9)^{* * *}$ & $11.1(7.3)^{\dagger \dagger \dagger}$ & $25.7(8.2)^{* * *+1 t}$ & 57.05 & & & 3,119 & .000 \\
\hline Craving for Cocaine (0-70) & - & - & $18.3(8.5)$ & $23.3(12.9)$ & & & -1.74 & 52 & .088 \\
\hline \multicolumn{10}{|l|}{ Tobacco } \\
\hline Smoking status $(\mathrm{y} / \mathrm{n})^{\mathrm{a}}$ & $22 / 18$ & $11 / 18$ & $19 / 11$ & $19 / 5$ & & 9.69 & & 3 & .021 \\
\hline Dependence $(y / n)^{b}$ & $9 / 31$ & $3 / 26$ & $15 / 15$ & $10 / 14$ & & 13.66 & & 3 & .003 \\
\hline Cigarettes per day & $5.3(7.5)$ & $2.7(5.7)$ & $11(12.1)^{\dagger \dagger}$ & $13(10.2)^{* *+\dagger \dagger}$ & 7.87 & & & 3,119 & .000 \\
\hline Years of use & $5.3(5.5)$ & $4.7(7.4)$ & $7.7(7)$ & $10.6(6.1)^{* \dagger \dagger}$ & 4.71 & & & 3,119 & .004 \\
\hline \multicolumn{10}{|l|}{ Alcohol } \\
\hline Alcohol use $(y / n)^{\mathrm{a}}$ & $39 / 1$ & $29 / 0$ & $30 / 0$ & $24 / 0$ & & 2.09 & & & .554 \\
\hline Dependence $(\mathrm{y} / \mathrm{n})^{\mathrm{c}}$ & $0 / 40$ & $1 / 28$ & $4 / 26$ & $2 / 22$ & & 6.26 & & 3 & .100 \\
\hline Grams per week ${ }^{\mathrm{a}}$ & $110.6(147.2)$ & $53.1(40.2)$ & $194.4(238.5)^{\dagger \dagger}$ & $177.2(146.7)^{\dagger}$ & 4.77 & & & 3,119 & .004 \\
\hline Years of use & $7.9(4.4)$ & $7.7(6.7)$ & $9.6(5.1)$ & $10.6(5.4)^{\dagger}$ & 1.91 & & & 3,119 & .131 \\
\hline \multicolumn{10}{|l|}{ Cocaine } \\
\hline Dependence $(\mathrm{y} / \mathrm{n})^{\mathrm{c}}$ & - & - & $6 / 24$ & $7 / 14$ & & 0.61 & & 1 & .528 \\
\hline Grams per week ${ }^{\mathrm{a}}$ & - & - & $2.3(3.1)$ & $2.1(3.1)$ & & & 0.27 & 52 & .789 \\
\hline Years of use & - & - & $6.3(5.1)$ & $7(4.9)$ & & & -0.53 & 52 & .599 \\
\hline Last consumption (days) & - & - & $22.3(33) n=30$ & $26.6(35.1) n=24$ & & & -.47 & 52 & .643 \\
\hline Cumulative dose (grams) & - & - & $1932.6(5169.8)$ & $3174.2(8456)$ & & & -0.67 & 52 & .509 \\
\hline Positive urine test ${ }^{\mathrm{d}}(\%)$ & $0(0)$ & $0(0)$ & $8(27.6)$ & $5(20.8)$ & & .32 & & 1 & .570 \\
\hline Hair analysis pg/mg & $0(0)$ & $0(0)$ & $5212.8(9195.6)$ & $5509.8(9517.2)$ & 7.47 & & & 3,118 & .000 \\
\hline \multicolumn{10}{|l|}{ Methylphenidate } \\
\hline Tablets per week ${ }^{\mathrm{a}, \mathrm{f}}$ & $0(0)$ & $15.9(18.4)$ & $0.9(2.9)^{\dagger \dagger \dagger}$ & $1.9(4)^{\dagger \dagger \dagger}$ & 20.17 & & & 3,119 & .000 \\
\hline Years of use & $0(0)$ & $2.6(3.7)$ & $0.1(0.3)^{\dagger \dagger \dagger}$ & $0.1(0.2)^{\dagger \dagger \dagger}$ & 15.44 & & & 3,119 & .000 \\
\hline Last consumption (days) & & $8.8(19.4) n=24$ & $48.3(53.7) \mathrm{n}=5$ & & & & -2.94 & 27 & .007 \\
\hline Cumulative dose $\mathrm{f}^{\mathrm{f}}$ & $0(0)$ & $2001(2672)$ & $36.8(124.9)^{\dagger \dagger \dagger}$ & $49.3(133.3)^{\dagger \dagger \dagger}$ & 17.12 & & & 3,119 & .000 \\
\hline
\end{tabular}


Urine toxicology MPH ng/ml

Urine tox. Ritalin acid ng/ml

Hair analysis pg/mg ${ }^{\mathrm{e}}$

\section{Cannabis}

Grams per week ${ }^{\mathrm{a}}$

Years of use

Cumulative dose (grams)

Last consumption (days)

Positive urine testing ${ }^{\mathrm{d}}(\%)$
$1.14(3.4) \mathrm{n}=27$

na

$0(0)$

$327.94(721.8) n=27$

191 (342.4)

$0.2(0.6)$

3.5 (4.2)

$919.8(4372.6)$

35 (43.4) $n=17$

$3(7.5)$

$$
\begin{gathered}
0.1(0.4) \\
2.7(4.3) \\
235.2(613.7)
\end{gathered}
$$

$27.8(29.7) \mathrm{n}=8$

$3(10.3)$ na

na

$15.8(77.8)$

$0.2(0.3)$

$6.6(5.1)^{\dagger}$

$972.3(1658.2)$

$25.2(29.3) n=22$

$3(10.3)$ na

na
8.84

$2.2(4.3)^{* * *+\dagger+1+t}$
$8.2(6.5)^{* *+1 \dagger}$
$1989.3(3303.2)$
$18(28.3) \mathrm{n}=18$

7.45

7.69

1.49

0.77

15.62
3

3,119

3, 119

3,119

3,61

3

Significant $p$-values are shown in bold. Statistical tests: ANOVA (all groups), $\chi 2$ test (all groups or cocaine user groups) for frequency data or independent $t$ test (two groups).

ADHD, attention-deficit/hyperactivity disorder; ADHD-SR, ADHD self-rating scale; CU+ADHD, cocaine users with ADHD; CU-ADHD, cocaine users without ADHD; MPH, methylphenidate.

Consumption per week, duration of use, and cumulative dose are averages within the total group.

Last consumption is an average only for persons who used the drug within the past 6 months. In this case, sample size (n) is shown.

${ }^{\text {a }}$ During the past 6 months.

${ }^{\mathrm{b}}$ Fagerström Test $>2$ points.

c According to DSM-IV criteria.

d For cut-offs, see the Supplementary Methods S2. One urine sample (CU-ADHD) was missing.

${ }^{\mathrm{e}}$ One hair sample (ADHD) is missing.

${ }^{\mathrm{f}}$ In 10 -mg tablets.

Sidak post-hoc tests vs. controls: $* p<.05, * * p<.01$; vs. ADHD $\uparrow p<.05, \dagger+p<.01, \uparrow \dagger \uparrow p<.001$; vs. CU-ADHD: $+\dagger+p<.001$ 


\section{FIGURE LEGENDS}

Figure 1: Mean z-scores and standard errors for the four cognitive domains and the global cognitive index

Values corrected for years of education and age. Sidak post-hoc tests vs. controls: $* * p<.01$, $* * * p<.001$; vs. ADHD: $\dagger \dagger p<.01, \dagger \dagger \dagger p<.001$; vs. CU-: $₫ p<.05$. Cohen's $d$ effect sizes for group comparisons vs. controls are shown.

Figure 2: Mean scores and standard errors for a) the global cognitive index (GCI), b) Theory-ofMind, and c) the Beck Depression Inventory (BDI) for all participants $(\mathrm{n}=123)$

Two-way analysis of covariance (ANCOVA) revealed summative effects of ADHD and cocaine use on cognition ( $p>$.05). However, these two factors show a significant interaction effect on Theory-ofMind and depressive symptoms (both $p<.05$ ), indicating a mutual reinforcement. ADHD = attentiondeficit/hyperactivity disorder, MASC = Movie for the Assessment of Social Cognition.

Figure 3: Mean sum score and standard errors for the Beck Depression Inventory

Values corrected for years of education and age. Sidak post-hoc tests vs. controls: $*^{* *} p<.001$; vs. ADHD: $\uparrow \uparrow \uparrow p<.001$; vs. CU: $\$ \uparrow t p<.001$. Cohen's $d$ effect sizes for group comparisons vs. controls are also shown. 


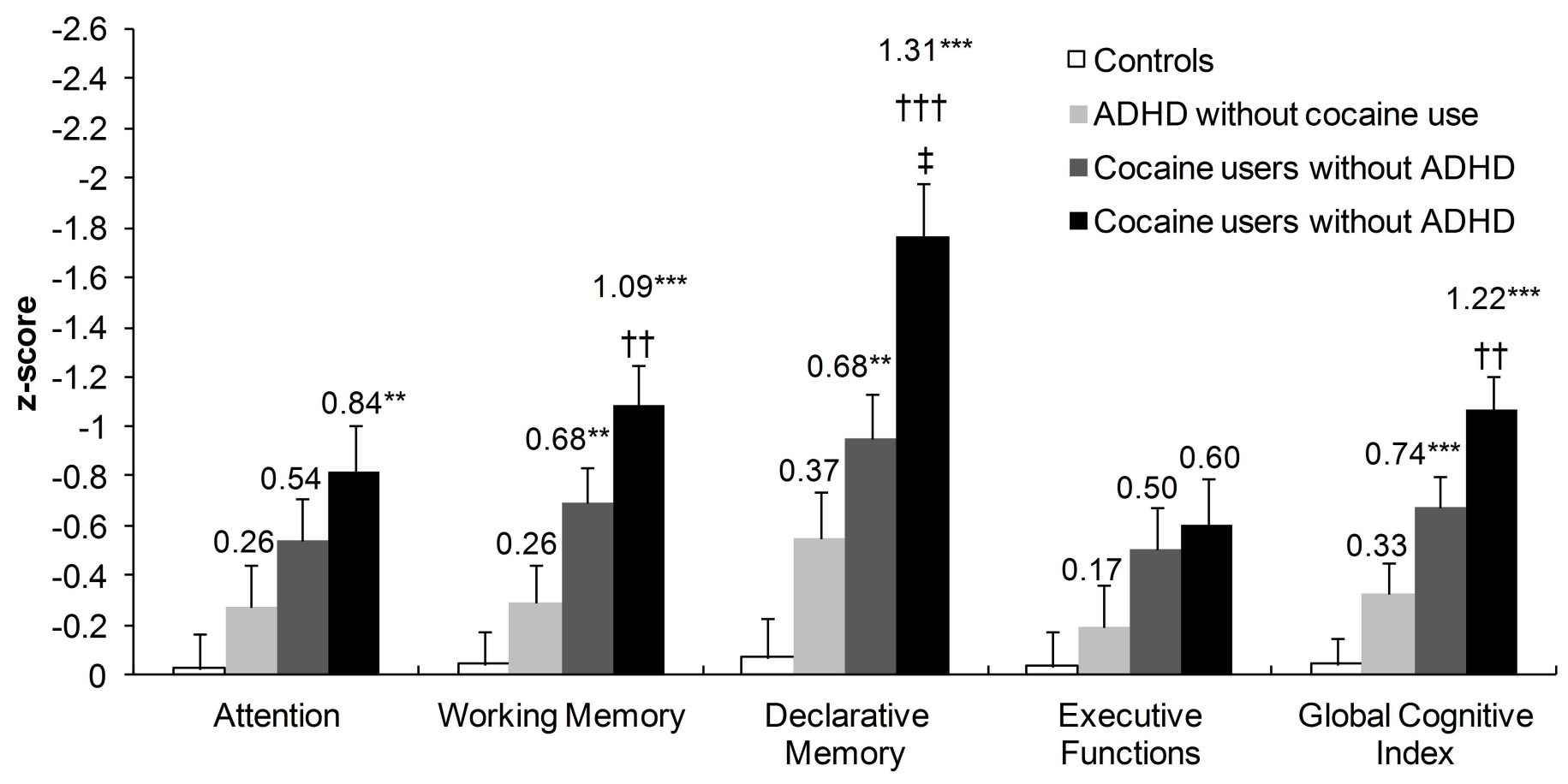



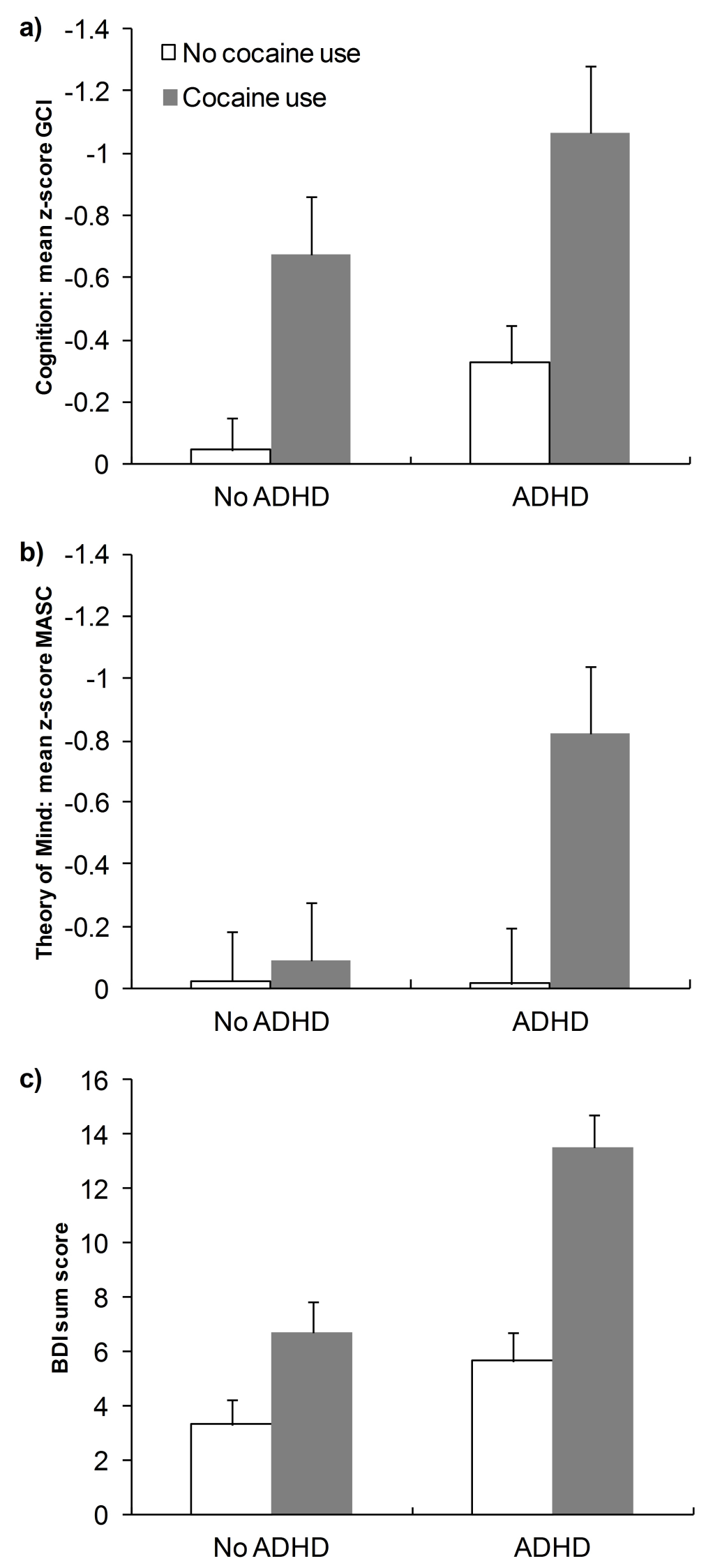


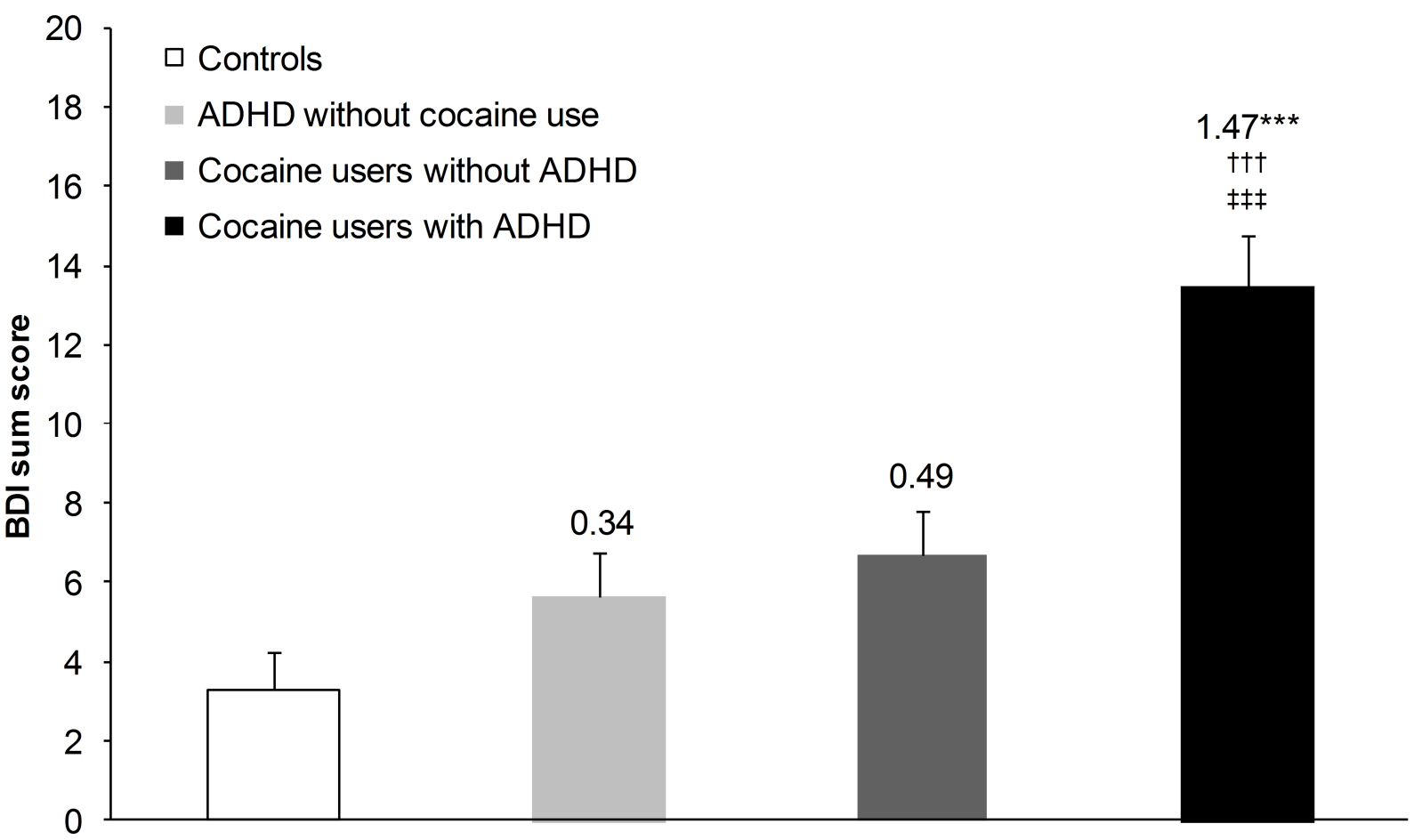

